\title{
ARTICLE
}

\section{Estimates of extreme solar particle event radiation exposures on Mars}

\author{
Lawrence W. Townsend*, Anne M. Adamczyk, Charles M. Werneth, Hanna M. Moussa and Jeremy P. Townsend
}

The University of Tennessee, Knoxville, Tennessee, 37996-2300 USA

\begin{abstract}
Estimates of effective doses and organ doses for male and female crew members are made for solar particle event proton environments comparable to several of the most significant solar particle events, which occurred in the second half of the $19^{\text {th }}$ century $(1864,1878,1894,1895$, and 1896). The incident proton energy distributions for these solar particle events are assumed to be similar to that of the November 1960 event, one of the most energetic of the modern space era. The crewmembers are assumed to be located at the mean surface elevation on Mars, at the lowest elevation on Mars in the Hellas Impact Basin, and on the summit of Olympus Mons, the highest surface elevation on Mars. The crewmembers are assumed to be shielded by the overlying carbon dioxide atmosphere of Mars, and locally shielded by a space suit, a surface landing spacecraft, or a surface habitat. These estimates are compared with current NASA Permissible Exposure Limits.
\end{abstract}

\section{Keywords: space radiation exposure; solar particle event; Mars; crew doses}

\section{Introduction}

Future missions to Mars may include human crews who might be exposed to potentially lethal or mission threatening solar particle events (SPEs). Thus, estimating exposures to plausible worst case events is important for evaluating shield requirements needed to protect human crews. Hypothetical worst case SPEs were previously evaluated [1-2] using fluences based on the nitrate concentration in ice core samples [3]. Recently, the validity of these ice core results has been called into question [4]. Others, however, have defended their validity [5]. Resolution of this issue remains to be decided. Nevertheless, in this work, the fluence of protons for the next five largest possible events (1864, $1878,1894,1895$, and 1896) in the ice core data, since 1859 and prior to the modern era of space exploration (1950's), will be used in combination with the proton energy spectrum from the November 1960 SPE, which is one of the largest events of the modern space era.

\section{Computational methods}

In this work, organ doses and effective doses, relevant for comparisons to the NASA permissible exposure limits (PELs) are calculated using NASA's On-Line Tool for the Assessment of Radiation in Space (OLTARIS) [6] for three possible Martian surface scenarios: a space suit $\left(0.3 \mathrm{~g} \mathrm{~cm}^{-2}\right.$ aluminum areal density), a surface landing spacecraft $\left(5 \mathrm{~g} \mathrm{~cm}^{-2}\right.$

*Corresponding author. Email: 1townsen@tennessee.edu aluminum areal density), or a surface habitat $\left(40 \mathrm{~g} \mathrm{~cm}^{-2}\right.$ aluminum areal density.

\subsection{Incident SPE spectra}

The modeled incident spectrum uses a Band function fit to the November 1960 SPE renormalized to the $>30$ $\mathrm{MeV}$ proton fluences from the ice core analyses for each event. These fluence values are displayed in Table 1.

Table 1. Integral fluences for the five events. Also displayed are the integral fluence values, $\mathrm{J}_{0}$, for each event.

\begin{tabular}{|c|c|c|}
\hline $\begin{array}{l}\text { Event } \\
\text { Year }\end{array}$ & 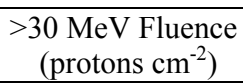 & $\begin{array}{c}J_{0} \\
\text { (protons cm }{ }^{-2} \text { ) }\end{array}$ \\
\hline 1864 & $7.00 \times 10^{9}$ & $6.39 \times 10^{9}$ \\
\hline 1878 & $5.00 \times 10^{9}$ & $4.57 \times 10^{9}$ \\
\hline 1894 & $7.70 \times 10^{9}$ & $7.03 \times 10^{9}$ \\
\hline 1895 & $1.11 \times 10^{10}$ & $1.01 \times 10^{10}$ \\
\hline 1896 & $8.00 \times 10^{9}$ & $7.31 \times 10^{9}$ \\
\hline
\end{tabular}

The Band function parameterization is given by [7]

$$
\Phi(>R)=\left\{\begin{array}{rr}
J_{0} R^{-\gamma_{1}} \exp \left(-\frac{R}{R_{0}}\right) \quad \text { for } R \leq\left(\gamma_{2}-\gamma_{1}\right) R_{0} \\
J_{0} R^{-\gamma_{2}}\left[\left(\gamma_{2}-\gamma_{1}\right) R_{0}\right]^{\left(\gamma_{2}-\gamma_{1}\right)} \exp \left(\gamma_{1}-\gamma_{2}\right) \\
\text { for }>R\left(\gamma_{2}-\gamma_{1}\right) R_{0}
\end{array}\right.
$$

Here, $\Phi$ is the proton fluence, $J_{0}$ is the total integral proton fluence, $R$ is the particle rigidity (momentum per unit charge), $R_{0}=0.321 \mathrm{GV}$ is the characteristic rigidity, 
and $\gamma_{1}=0.584$ and $\gamma_{2}=5.04$ are spectral indices. Values for $\mathrm{J}_{0}$ are listed in Table 1 .

\subsection{Particle transport}

Transport methods used in OLTARIS are based on HZETRN2010 [8], the latest version of the deterministic space radiation transport code developed at NASA Langley Research Center. In this work, HZETRN models the transport of all incident charged protons and their nuclear reaction products (protons, neutrons, deuterons, tritons, helions, and alpha particles). The database of secondary nuclear reaction products for HZETRN2010 is provided by the nuclear fragmentation model NUCFRG3 [9]. In this application, the particles are transported through $300 \mathrm{~g} \mathrm{~cm}^{-2}$ of Mars' carbon dioxide atmosphere, followed by transport through the appropriate aluminum shielding $\left(0.3,5\right.$ or $\left.40 \mathrm{~g} \mathrm{~cm}^{-2}\right)$, and then transport through human tissue. The resulting doses are folded with the computerized anatomical male (CAM) and computerized anatomical female (CAF) models [10-11] to estimate organ doses and effective doses.

\subsection{Radiation exposure quantities and limits}

\subsubsection{Organ doses and limits}

Table 2. NASA PELs for short term or career non-cancer effects. N/A means not applicable.

\begin{tabular}{|c|c|c|c|}
\hline Organ & $\begin{array}{c}30 \text { day } \\
\text { Limit } \\
(\mathrm{cGy-Eq})\end{array}$ & $\begin{array}{c}1 \text { year } \\
\text { Limit } \\
(\mathrm{cGy-Eq})\end{array}$ & $\begin{array}{c}\text { Career } \\
\text { Limit } \\
(\mathrm{cGy-Eq})\end{array}$ \\
\hline Lens & 100 & 200 & 400 \\
\hline Skin & 150 & 300 & 400 \\
\hline BFO & 25 & 50 & N/A \\
\hline Heart & 25 & 50 & 100 \\
\hline CNS & 50 & 100 & 150 \\
\hline
\end{tabular}

NASA radiation limits for short term (acute) radiation exposures are presented in Table 2, which lists permissible exposure limits (PELs) for thirty days, one year, and career for non-cancer radiation effects [12]. Organs considered are the eye lens, skin, blood forming organs (BFO), heart, and central nervous system (CNS). For comparison to the PELs, organ doses are converted from units of centigray (cGy) to units of centigray-equivalent (cGy-Eq) using a Relative Biological Effectiveness (RBE) factor as specified in Ref. [13]. This is accomplished using Eq. (2) where, for protons, $\mathrm{RBE}=1.5$.

$$
D[c G y-E q]=D[c G y] \times R B E
$$

\subsubsection{Effective dose and limits}

Effective dose $(\mathrm{E})$, in units of centiSievert $(\mathrm{cSv})$, is calculated using

$$
E=\sum_{T} w_{T} H_{T},
$$

where $w_{T}$ is the tissue weighting factor obtained from Ref. [15]. The organ dose equivalent $H_{T}$, in Eq. (3), is in units of $\mathrm{cSv}$ and is calculated in OLTARIS from the product of the radiation quality factor, $Q$ [14] and organ dose, D. NASA's career effective dose limits [13], shown below in Table 3, are established to reduce the probability of developing a fatal cancer to $3 \%$ with $95 \%$ confidence.

Table 3. Career exposure limits as a function of age at first exposure for a mission not exceeding one year in duration.

\begin{tabular}{|c|c|c|}
\hline \multirow{2}{*}{$\begin{array}{c}\text { Age } \\
\text { (years) }\end{array}$} & \multicolumn{2}{|c|}{$\begin{array}{c}\text { Effective Dose } \\
\text { (cSv) }\end{array}$} \\
\cline { 2 - 3 } & Male & Female \\
\hline 25 & 52 & 37 \\
\hline 30 & 62 & 47 \\
\hline 35 & 72 & 55 \\
\hline 40 & 80 & 62 \\
\hline 45 & 95 & 75 \\
\hline 50 & 115 & 92 \\
\hline 55 & 147 & 112 \\
\hline
\end{tabular}

\subsection{Radiation exposure scenarios}

Organ doses and effective doses, for male (CAM) and female (CAF) crew members shielded by a space suit, a surface landing spacecraft, and a surface habitat are calculated for locations at the top of Olympus Mons $\left(+25 \mathrm{~km}\right.$ altitude - the highest point on Mars, $2.2 \mathrm{~g} \mathrm{~cm}^{-2}$ $\left.\mathrm{CO}_{2}\right)$, the mean surface elevation datum $(0 \mathrm{~km}$ altitude, $\left.16.7 \mathrm{~g} \mathrm{~cm}^{-2} \mathrm{CO}_{2}\right)$, and in the Hellas Impact Basin $(-7 \mathrm{~km}$ altitude - the lowest point on Mars, $\left.30.5 \mathrm{~g} \mathrm{~cm}^{-2} \mathrm{CO}_{2}\right)$. For each location, incident SPE protons arrive from all angles between 0 and 90 degrees with respect to the local zenith and are averaged over all particle arrival path length areal densities.

\section{Organ dose and effective dose results}

Organ doses and effective doses are presented below in Tables 4 - 9. In Tables 4 - 8 organ doses that exceed the PELs are indicated in bold. It is clear that nearly all organ doses at the summit of Olympus Mons, shielded only by a space suit, exceed dose limits. Also, BFO, CNS and heart dose limits may be exceeded for some events within a lander located at the summit. No limits are exceeded within a habitat or for any shield configuration at or lower than the mean surface elevation. From Table 9, effective dose limits are exceeded for males under the age of 45 and females under the age of 50 shielded by only a space suit.

\section{Concluding remarks}

Radiation exposures on Mars for a variety of hypothetical, large SPEs have been presented. In nearly all scenarios, only thinly-shielded crews on the summit of Olympus Mons appear to exceed exposure limits. As expected, the 1895 event, which had the highest proton fluence, yielded the largest exposures. 
Table 4. Skin dose as a function of event, altitude, and aluminum shielding for male (CAM) and female (CAF) crew members. Skin doses for the 1878 event are all below the PELs and are not shown.

\begin{tabular}{|c|c|c|c|c|c|c|c|c|c|c|}
\hline \multicolumn{11}{|c|}{ Skin Dose (cGy-Eq) } \\
\hline \multirow{3}{*}{$\begin{array}{l}\text { Event } \\
\text { Year }\end{array}$} & \multirow{3}{*}{$\begin{array}{c}\text { Human } \\
\text { Body } \\
\text { Model }\end{array}$} & \multirow{2}{*}{\multicolumn{3}{|c|}{$\frac{0.3 \mathrm{~g} \mathrm{~cm}^{-2} \mathrm{Al} \text { Shield }}{\text { Elevation }}$}} & \multirow{2}{*}{\multicolumn{3}{|c|}{$\begin{array}{c}5 \mathrm{~g} \mathrm{~cm}^{-2} \mathrm{Al} \text { Shield } \\
\text { Elevation }\end{array}$}} & \multicolumn{3}{|c|}{$40 \mathrm{~g} \mathrm{~cm}^{-2} \mathrm{Al}$ Shield } \\
\hline & & & & & & & & & evation & \\
\hline & & $+25 \mathrm{~km}$ & $0 \mathrm{~km}$ & $-7 \mathrm{~km}$ & $+25 \mathrm{~km}$ & $0 \mathrm{~km}$ & $-7 \mathrm{~km}$ & $+25 \mathrm{~km}$ & $0 \mathrm{~km}$ & $-7 \mathrm{~km}$ \\
\hline \multirow[t]{2}{*}{1864} & CAM & 154 & 11 & 4 & 70 & 9 & 4 & 10 & 3 & 2 \\
\hline & CAF & 153 & 11 & 4 & 70 & 9 & 4 & 10 & 3 & 2 \\
\hline \multirow[t]{2}{*}{1894} & CAM & 158 & 12 & 5 & 72 & 9 & 4 & 10 & 3 & 2 \\
\hline & CAF & 157 & 12 & 5 & 72 & 9 & 4 & 10 & 4 & 2 \\
\hline \multirow[t]{2}{*}{1895} & CAM & 244 & 18 & 7 & 111 & 14 & 6 & 16 & 5 & 3 \\
\hline & CAF & 243 & 18 & 7 & 111 & 15 & 6 & 16 & 5 & 3 \\
\hline \multirow[t]{2}{*}{1896} & CAM & 176 & 13 & 5 & 80 & 10 & 4 & 12 & 4 & 2 \\
\hline & CAF & 175 & 13 & 5 & 80 & 11 & 4 & 12 & 4 & 2 \\
\hline
\end{tabular}

Table 5. Eye lens dose as a function of event, altitude, and aluminum shielding for male (CAM) and female (CAF) crew member. Eye lens doses for the 1878 event are all below the PELs and are not shown.

\begin{tabular}{|c|c|c|c|c|c|c|c|c|c|c|}
\hline \multicolumn{11}{|c|}{ Eye Lens Dose (cGy-Eq) } \\
\hline \multirow{3}{*}{$\begin{array}{l}\text { Event } \\
\text { Year }\end{array}$} & \multirow{3}{*}{$\begin{array}{c}\text { Human } \\
\text { Body } \\
\text { Model }\end{array}$} & \multirow{2}{*}{\multicolumn{3}{|c|}{$\frac{0.3 \mathrm{~g} \mathrm{~cm}^{-2} \mathrm{Al} \text { Shield }}{\text { Elevation }}$}} & \multirow{2}{*}{\multicolumn{3}{|c|}{$\frac{5 \mathrm{~g} \mathrm{~cm}^{-2} \mathrm{Al} \mathrm{Shield}}{\text { Elevation }}$}} & \multirow{2}{*}{\multicolumn{3}{|c|}{$\frac{40 \mathrm{~g} \mathrm{~cm}^{-2} \mathrm{Al} \text { Shield }}{\text { Elevation }}$}} \\
\hline & & & & & & & & & & \\
\hline & & $+25 \mathrm{~km}$ & $0 \mathrm{~km}$ & $-7 \mathrm{~km}$ & $+25 \mathrm{~km}$ & $0 \mathrm{~km}$ & $-7 \mathrm{~km}$ & $+25 \mathrm{~km}$ & $0 \mathrm{~km}$ & $-7 \mathrm{~km}$ \\
\hline \multirow[t]{2}{*}{1864} & CAM & 101 & 11 & 4 & 58 & 9 & 4 & 10 & 3 & 2 \\
\hline & CAF & 102 & 10 & 4 & 59 & 9 & 4 & 10 & 4 & 2 \\
\hline \multirow[t]{2}{*}{1894} & CAM & 103 & 11 & 5 & 59 & 9 & 4 & 10 & 4 & 2 \\
\hline & $\mathrm{CAF}$ & 105 & 10 & 5 & 60 & 9 & 4 & 10 & 4 & 2 \\
\hline \multirow[t]{2}{*}{1895} & CAM & 159 & 17 & 7 & 91 & 14 & 6 & 16 & 5 & 3 \\
\hline & CAF & 162 & 16 & 7 & 93 & 14 & 6 & 16 & 6 & 3 \\
\hline \multirow[t]{2}{*}{1896} & CAM & 115 & 12 & 5 & 66 & 10 & 4 & 12 & 4 & 2 \\
\hline & $\mathrm{CAF}$ & 117 & 11 & 5 & 6 & 10 & 4 & 12 & 4 & 2 \\
\hline
\end{tabular}

Table 6. BFO dose as a function of event, altitude, and aluminum shielding for male (CAM) and female (CAF) crew members.

\begin{tabular}{|c|c|c|c|c|c|c|c|c|c|c|}
\hline \multicolumn{11}{|c|}{ Blood Forming Organ Dose (cGy-Eq) } \\
\hline \multirow{3}{*}{$\begin{array}{l}\text { Event } \\
\text { Year }\end{array}$} & \multirow{3}{*}{$\begin{array}{l}\text { Human } \\
\text { Body } \\
\text { Model }\end{array}$} & \multirow{2}{*}{\multicolumn{3}{|c|}{$\frac{0.3 \mathrm{~g} \mathrm{~cm}^{-2} \mathrm{Al} \mathrm{Shield}}{\text { Elevation }}$}} & \multirow{2}{*}{\multicolumn{3}{|c|}{$\begin{array}{c}5 \mathrm{~g} \mathrm{~cm}^{-2} \mathrm{Al} \text { Shield } \\
\text { Elevation }\end{array}$}} & \multirow{2}{*}{\multicolumn{3}{|c|}{$\frac{40 \mathrm{~g} \mathrm{~cm}^{-2} \mathrm{Al} \text { Shield }}{\text { Elevation }}$}} \\
\hline & & & & & & & & & & \\
\hline & & $+25 \mathrm{~km}$ & $0 \mathrm{~km}$ & $-7 \mathrm{~km}$ & $+25 \mathrm{~km}$ & $0 \mathrm{~km}$ & $-7 \mathrm{~km}$ & $+25 \mathrm{~km}$ & $0 \mathrm{~km}$ & $-7 \mathrm{~km}$ \\
\hline \multirow[t]{2}{*}{1864} & CAM & 47 & 8 & 4 & 33 & 7 & 3 & 8 & 3 & 2 \\
\hline & CAF & 50 & 7 & 4 & 34 & 7 & 3 & 8 & 3 & 2 \\
\hline \multirow[t]{2}{*}{1878} & CAM & 34 & 6 & 3 & 24 & 5 & 2 & 6 & 2 & 1 \\
\hline & CAF & 36 & 5 & 3 & 25 & 5 & 2 & 6 & 2 & 1 \\
\hline \multirow[t]{2}{*}{1894} & CAM & 49 & 8 & 4 & 34 & 7 & 3 & 8 & 3 & 2 \\
\hline & CAF & 51 & 8 & 4 & 35 & 7 & 3 & 8 & 3 & 2 \\
\hline \multirow[t]{2}{*}{1895} & CAM & 75 & 12 & 6 & 52 & 11 & 5 & 12 & 5 & 3 \\
\hline & CAF & 79 & 12 & 6 & 54 & 11 & 5 & 13 & 5 & 3 \\
\hline \multirow[t]{2}{*}{1896} & CAM & 54 & 9 & 4 & 38 & 8 & 4 & 9 & 3 & 2 \\
\hline & CAF & 57 & 8 & 4 & 39 & 8 & 4 & 9 & 3 & 2 \\
\hline
\end{tabular}

Table 7. CNS dose as a function of event, altitude, and aluminum shielding for male (CAM) and female (CAF) crew members. CNS doses for the 1878 event are all below the PELs and are not shown.

\begin{tabular}{|c|c|c|c|c|c|c|c|c|c|c|}
\hline \multicolumn{11}{|c|}{ CNS Dose (cGy-Eq) } \\
\hline \multirow{3}{*}{$\begin{array}{l}\text { Event } \\
\text { Year }\end{array}$} & \multirow{3}{*}{$\begin{array}{c}\text { Human } \\
\text { Body } \\
\text { Model }\end{array}$} & \multirow{2}{*}{\multicolumn{3}{|c|}{$\begin{array}{c}0.3 \mathrm{~g} \mathrm{~cm}^{-2} \mathrm{Al} \mathrm{Shield} \\
\text { Elevation }\end{array}$}} & \multirow{2}{*}{\multicolumn{3}{|c|}{$\begin{array}{c}5 \mathrm{~g} \mathrm{~cm}^{-2} \mathrm{Al} \text { Shield } \\
\text { Elevation }\end{array}$}} & \multirow{2}{*}{\multicolumn{3}{|c|}{$\frac{40 \mathrm{~g} \mathrm{~cm}^{-2} \mathrm{Al} \mathrm{Shield}}{\text { Elevation }}$}} \\
\hline & & & & & & & & & & \\
\hline & & $+25 \mathrm{~km}$ & $0 \mathrm{~km}$ & $-7 \mathrm{~km}$ & $+25 \mathrm{~km}$ & $0 \mathrm{~km}$ & $-7 \mathrm{~km}$ & $+25 \mathrm{~km}$ & $0 \mathrm{~km}$ & $-7 \mathrm{~km}$ \\
\hline \multirow[t]{2}{*}{1864} & CAM & 50 & 9 & 4 & 36 & 7 & 3 & 9 & 3 & 2 \\
\hline & CAF & 54 & 9 & 4 & 38 & 8 & 4 & 9 & 3 & 2 \\
\hline \multirow[t]{2}{*}{1894} & CAM & 51 & 9 & 4 & 37 & 8 & 4 & 9 & 3 & 2 \\
\hline & $\mathrm{CAF}$ & 55 & 9 & 4 & 39 & 8 & 4 & 9 & 3 & 2 \\
\hline \multirow[t]{2}{*}{1895} & CAM & 79 & 14 & 6 & 57 & 12 & 5 & 13 & 5 & 3 \\
\hline & CAF & 85 & 14 & 6 & 60 & 12 & 6 & 14 & 5 & 3 \\
\hline \multirow[t]{2}{*}{1896} & CAM & 57 & 10 & 4 & 41 & 8 & 4 & 10 & 4 & 2 \\
\hline & CAF & 61 & 10 & 4 & 44 & 9 & 4 & 10 & 4 & 2 \\
\hline
\end{tabular}


Table 8. Heart dose as a function of event, altitude, and aluminum shielding for male (CAM) and female (CAF) crew members.

\begin{tabular}{|c|c|c|c|c|c|c|c|c|c|c|}
\hline \multicolumn{11}{|c|}{ Heart Dose (cGy-Eq) } \\
\hline \multirow{3}{*}{$\begin{array}{l}\text { Event } \\
\text { Year }\end{array}$} & \multirow{3}{*}{$\begin{array}{l}\text { Human } \\
\text { Body } \\
\text { Model }\end{array}$} & \multirow{2}{*}{\multicolumn{3}{|c|}{$\frac{0.3 \mathrm{~g} \mathrm{~cm}^{-2} \mathrm{Al} \text { Shield }}{\text { Elevation }}$}} & \multirow{2}{*}{\multicolumn{3}{|c|}{$\frac{5 \mathrm{~g} \mathrm{~cm}^{-2} \mathrm{Al} \text { Shield }}{\text { Elevation }}$}} & \multicolumn{3}{|c|}{$40 \mathrm{~g} \mathrm{~cm}^{-2} \mathrm{Al}$ Shield } \\
\hline & & & & & & & & & evation & \\
\hline & & $+25 \mathrm{~km}$ & $0 \mathrm{~km}$ & $-7 \mathrm{~km}$ & $+25 \mathrm{~km}$ & $0 \mathrm{~km}$ & $-7 \mathrm{~km}$ & $+25 \mathrm{~km}$ & $0 \mathrm{~km}$ & $-7 \mathrm{~km}$ \\
\hline \multirow[t]{2}{*}{1864} & CAM & 36 & 7 & 3 & 27 & 6 & 3 & 7 & 3 & 2 \\
\hline & CAF & 38 & 7 & 3 & 28 & 6 & 3 & 7 & 3 & 2 \\
\hline \multirow[t]{2}{*}{1878} & CAM & 26 & 5 & 2 & 19 & 4 & 2 & 5 & 2 & 1 \\
\hline & $\mathrm{CAF}$ & 27 & 5 & 2 & 20 & 5 & 2 & 5 & 2 & 1 \\
\hline \multirow[t]{2}{*}{1894} & CAM & 37 & 7 & 3 & 28 & 6 & 3 & 7 & 3 & 2 \\
\hline & CAF & 39 & 8 & 3 & 29 & 7 & 3 & 8 & 3 & 2 \\
\hline \multirow[t]{2}{*}{1895} & CAM & 57 & 11 & 5 & 43 & 10 & 5 & 11 & 4 & 3 \\
\hline & CAF & 60 & 12 & 5 & 45 & 10 & 5 & 12 & 4 & 3 \\
\hline \multirow[t]{2}{*}{1896} & CAM & 41 & 8 & 4 & 31 & 7 & 3 & 8 & 3 & 2 \\
\hline & $\mathrm{CAF}$ & 43 & 8 & 4 & 32 & 7 & 3 & 8 & 3 & 2 \\
\hline
\end{tabular}

Table 9. Effective dose as a function of event, altitude, and aluminum shielding for male (CAM) and female (CAF) crew members.

\begin{tabular}{|c|c|c|c|c|c|c|c|c|c|c|}
\hline \multicolumn{11}{|c|}{ Effective Dose (cSv) } \\
\hline \multirow{3}{*}{$\begin{array}{l}\text { Event } \\
\text { Year }\end{array}$} & \multirow{3}{*}{$\begin{array}{c}\text { Human } \\
\text { Body } \\
\text { Model }\end{array}$} & \multirow{2}{*}{\multicolumn{3}{|c|}{$\frac{0.3 \mathrm{~g} \mathrm{~cm}^{-2} \mathrm{Al} \mathrm{Shield}}{\text { Elevation }}$}} & \multirow{2}{*}{\multicolumn{3}{|c|}{$\begin{array}{c}5 \mathrm{~g} \mathrm{~cm}^{-2} \mathrm{Al} \text { Shield } \\
\text { Elevation }\end{array}$}} & \multirow{2}{*}{\multicolumn{3}{|c|}{$\frac{40 \mathrm{~g} \mathrm{~cm}^{-2} \mathrm{Al} \text { Shield }}{\text { Elevation }}$}} \\
\hline & & & & & & & & & & \\
\hline & & $+25 \mathrm{~km}$ & $0 \mathrm{~km}$ & $-7 \mathrm{~km}$ & $+25 \mathrm{~km}$ & $0 \mathrm{~km}$ & $-7 \mathrm{~km}$ & $+25 \mathrm{~km}$ & $0 \mathrm{~km}$ & $-7 \mathrm{~km}$ \\
\hline \multirow[t]{2}{*}{1864} & CAM & 52 & 10 & 5 & 35 & 9 & 5 & 10 & 5 & 3 \\
\hline & $\mathrm{CAF}$ & 49 & 10 & 5 & 34 & 9 & 5 & 10 & 5 & 3 \\
\hline \multirow[t]{2}{*}{1878} & CAM & 37 & 7 & 4 & 25 & 6 & 3 & 7 & 3 & 2 \\
\hline & $\mathrm{CAF}$ & 35 & 7 & 4 & 25 & 6 & 3 & 7 & 3 & 2 \\
\hline \multirow[t]{2}{*}{1894} & CAM & 54 & 10 & 5 & 36 & 9 & 5 & 11 & 5 & 3 \\
\hline & CAF & 51 & 10 & 5 & 35 & 9 & 5 & 11 & 5 & 3 \\
\hline \multirow[t]{2}{*}{1895} & CAM & 83 & 15 & 8 & 56 & 14 & 7 & 17 & 8 & 5 \\
\hline & $\mathrm{CAF}$ & 78 & 15 & 8 & 55 & 14 & 8 & 17 & 8 & 5 \\
\hline \multirow[t]{2}{*}{1896} & CAM & 60 & 11 & 6 & 40 & 10 & 5 & 12 & 6 & 4 \\
\hline & CAF & 56 & 11 & 6 & 39 & 10 & 5 & 12 & 5 & 4 \\
\hline
\end{tabular}

\section{References}

[1] L. Townsend, D. Stephens, J. Hoff, E. Zapp, H. Moussa, T. Miller, C. Campbell and T. Nichols, The Carrington event: possible doses to crews in space from a comparable event, Adv. Space. Res. 38 (2006), pp. 226-231.

[2] L. Townsend, M. PourArsalan, M. Hall, J. Anderson, S. Bhatt, N. DeLauder and A. Adamczyk, Estimates of Carrington-class solar particle event radiation exposures on Mars, Acta Astronautica 69 (2011), pp. 397-405.

[3] K. McCracken, G. Dreschhoff, D. Smart and M. Shea, Solar cosmic ray events for the period 1561-1994 1. Identification in polar ice, 1561-1950, J. Geophys. Res. 106 (2001), pp. 585-598.

[4] E. Wolff, M. Bigler, M. Curran, J. Dibb, M. Frey, M. Legrand and J. McConnell, The Carrington event not observed in most ice core nitrate records, Geophys. Res. Lett. 39, L08503 (2012),

[5] D. Smart and M. Shea, Extreme solar proton events that are possible $\mathrm{NO}_{\mathrm{y}}$ sources with residuals in polar ice, Extreme Space Weather Events Workshop, Boulder, Colorado, (2012).

[6] R. Singleterry, S. Blattnig, M. Clowdsley, G. Qualls, C. Sandridge, L. Simonsen, T. Slaba, S. Walker, F. Badavi, J. Spangler, A. Aumann, E.
Zapp, R. Rutledge, K. Lee, R. Norman and J. Norbury, OLTARIS: On-line tool for the assessment of radiation in space, Acta Astronautica 68 (2011), pp. 1086-1097.

[7] A.J. Tylka, W. Dietrich and W. Atwell, Band function representations of solar proton spectra in ground-level events, Paper F24-0004-10, in COSPAR XXXVIII Scientific Assembly, Bremen, Germany, July 18-25, 2010.

[8] T. Slaba, S. Blattnig and F. Badavi, Faster and more accurate transport procedures for HZETRN, J. Comp. Phys. 229 (2010), pp. 9397-9417.

[9] A. Adamczyk, R. Norman, S. Sriprisan, L. Townsend, J. Norbury, S. Blattnig and T. Slaba, NUCFRG3: Light ion improvements to the nuclear fragmentation model, Nuc. Instrum. Meth. Phys. A 678 (2012), pp. 21-32.

[10]M. Billings and W. Yucker, The Computerized Anatomical Man (CAM) Model, McDonnell Douglas Company Report MDC-G465, (1973).

[11]W. Yucker and S. Huston, The Computerized Anatomical Female, McDonnell Douglas Company Report MDC-6107, (1990).

[12]NASA, Space Flight Human System Standard Volume 1: Crew Health, NASA-STD-3001, (2009). 
[13] National Council on Radiation Protection and Measurements (NCRP), Radiation Protection Guidance for Activities in Low Earth Orbit, NCRP Report No. 132, (2000).
[14] International Commission on Radiological Protection (ICRP), The 1990 Recommendations of the International Commission on Radiological Protection, ICRP Publication 60, (1991). 